Article

\title{
Eucalyptol: A Bio-Based Solvent for the Synthesis of $O, S, N$-Heterocycles. Application to Hiyama Coupling, Cyanation, and Multicomponent Reactions
}

\author{
Joana F. Campos (D), Véronique Ferreira and Sabine Berteina-Raboin *(D)
}

\begin{abstract}
Institute of Organic and Analytical Chemistry (ICOA), University of Orleans, UMR-CNRS 7311, BP 6759, Rue de Chartres, CEDEX2, 45067 Orleans, France; joana-filomena.mimoso-silva-de-campos@univ-orleans.fr (J.F.C.); veronique.ferreira@univ-orleans.fr (V.F.)

* Correspondence: sabine.berteina-raboin@univ-orleans.fr
\end{abstract}

Citation: Campos, J.F.; Ferreira, V.; Berteina-Raboin, S. Eucalyptol: A Bio-Based Solvent for the Synthesis of $\mathrm{O}, \mathrm{S}, \mathrm{N}$-Heterocycles. Application to Hiyama Coupling, Cyanation, and Multicomponent Reactions. Catalysts 2021, 11, 222. https://doi.org/ $10.3390 /$ catal11020222

Academic Editor: Laura Antonella Aronica

Received: 20 January 2021

Accepted: 4 February 2021

Published: 8 February 2021

Publisher's Note: MDPI stays neutral with regard to jurisdictional claims in published maps and institutional affiliations.

Copyright: (c) 2021 by the authors. Licensee MDPI, Basel, Switzerland. This article is an open access article distributed under the terms and conditions of the Creative Commons Attribution (CC BY) license (https:// creativecommons.org/licenses/by/ $4.0 /)$.

\begin{abstract}
We report here the use of eucalyptol as a bio-based solvent for Hiyama coupling, cyanation, and multicomponent reactions on $\mathrm{O}, \mathrm{S}, \mathrm{N}$-heterocycles. These heterocycles were chosen as targets or as starting materials given their biological potential; they play an important role in therapeutically active compounds. Once again, eucalyptol proved to be a credible and sustainable alternative to common solvents.
\end{abstract}

Keywords: eucalyptol; sustainable chemistry; $\mathrm{O}, \mathrm{S}, \mathrm{N}$-Heterocycles; Hiyama coupling; cyanation reaction; multicomponent reaction

\section{Introduction}

The solvents used in chemistry are a fundamental element of the environmental performance of processes in industrial and academic laboratories. Their influence on costs, safety, and health cannot be neglected. Even if solvent-free reactions are possible to a certain extent, they are not applicable to a large spectrum of chemical reactions and starting materials, and they may impair overall yield and product purity. Equally important, multiphasic reactions involving solid catalysts and gaseous and/or liquid reagents, which are common practice in the petrochemical and refining industry, are not easily transposable to pharmaceutical active ingredients or fine chemical syntheses.

Solvents are the most abundant constituents of chemical transformations, so acting thereon and replacing standard solvents with safer alternatives can have a great ecological impact. Nitrogen heterocyclic compounds represent an important class of compounds in the pharmaceutical industry. Therefore, it is important to provide new methods and greener approaches for their synthesis [1-7].

Pursuing our objective of developing new practices in the synthesis of heterocycles containing oxygen, sulfur, and nitrogen [8-17], we explored the potential of eucalyptol [15] (Figures 1 and 2) as solvent in Hiyama coupling, cyanation, and multicomponent reactions.

Eucalyptol or 1,8-cineole (Figure 2) is a saturated oxygenated terpene that is widely distributed in some plants and their essential oil fractions, and depending on the species, it is contained in up to $90 \%$ in eucalyptus' essential oils isolated from fresh foliage. Its use as a solvent is also very interesting from an environmental point of view, since in addition to the fact that this solvent is recyclable by simple distillation, it comes from the waste (leaves) of the paper and wood industry, which cultivates eucalyptus trees because of their rapid growth (7 to 10 years). 


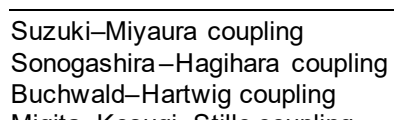

Migita-Kosugi-Stille coupling

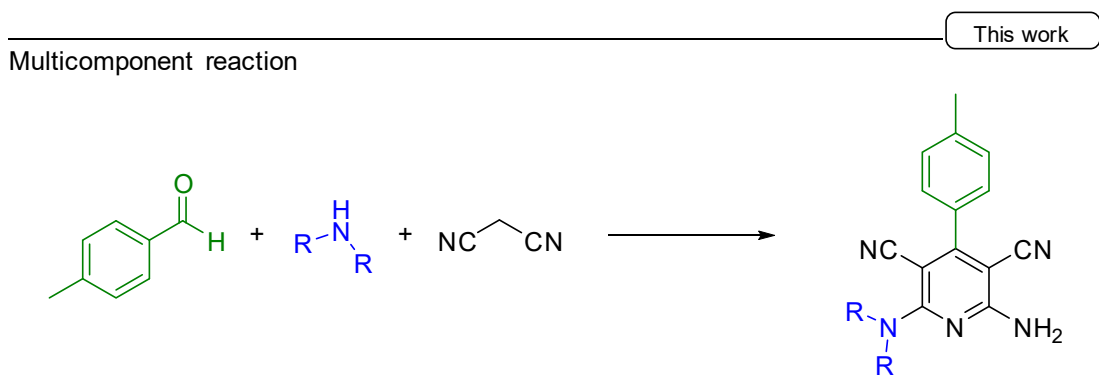

Cyanation

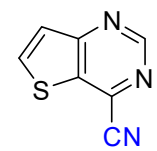<smiles>N#Cc1ccnc2ccsc12</smiles><smiles>N#Cc1c(-c2ccccc2)sc2nccnc12</smiles>

Hiyama Coupling<smiles>Clc1ccnc2ccsc12</smiles><smiles>[R][Si]1(C)CC1C</smiles><smiles>[R]c1ccnc2ccsc12</smiles>

Figure 1. Eucalyptol as bio-based solvent: application to Hiyama coupling, cyanation and multicomponent reactions.

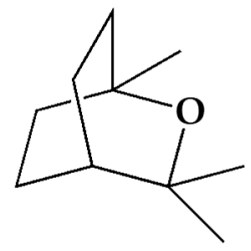

\section{Eucalyptol}

$\mathrm{C}_{10} \mathrm{H}_{18} \mathrm{O}$

bp : $176-177^{\circ} \mathrm{C}$

d : 0.921

$\varepsilon: 4.84$

CAS 470-82-6

Figure 2. Structure and data of Eucalyptol. Synonyms: 1,8-Cineole, 1,3,3-trimethyl-2oxabicyclo[2.2.2]octane.

\section{Results and Discussion}

\subsection{Multicomponent Reaction}

While the chemistry community has made significant efforts toward identifying greener processes that minimize the quantity of catalysts or using multicomponent reactions and one-pot processes, solvents remain a major portion of the environmental performance of a process and have an influence on safety and health [18-21]. One of the goals of the present study was to assess the potential of associating a multicomponent reaction with a more eco-compatible solvent. The class of molecules chosen for synthesis was highly functionalized pyridines [22]. In this specific case, to the best of our knowledge, only three teams have reported their synthesis; however, the reactions were performed using conventional solvents, namely chloroform [23], ethanol [24], and methanol [25]. After ascertaining the most widely used reaction conditions and stoichiometry, we performed the reactions using eucalyptol as solvent (Table 1). 
Table 1. Optimization of the multicomponent reaction.

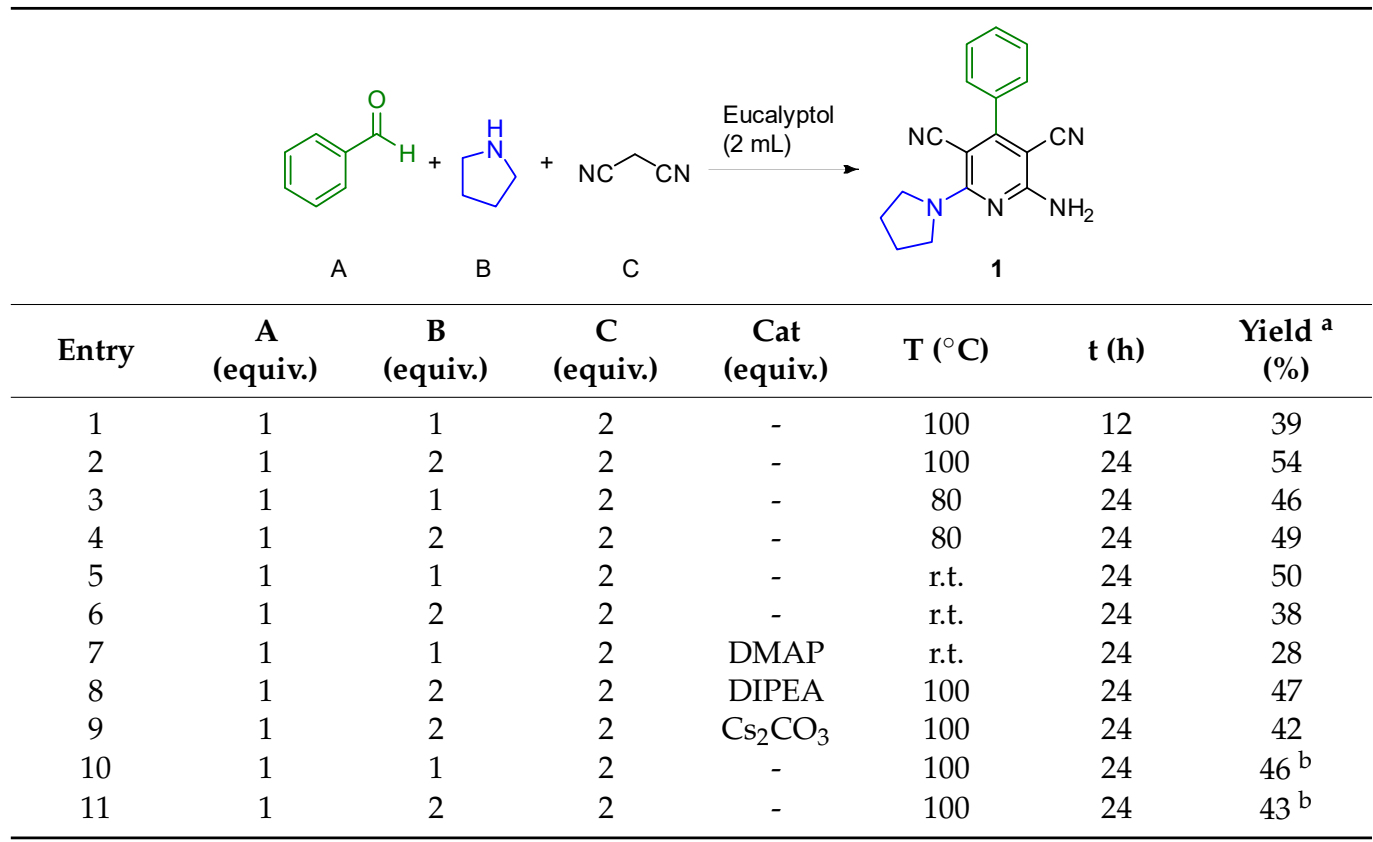

a isolated yield after purification via flash chromatography. ${ }^{\mathrm{b}}$ reaction performed in $\mathrm{MeOH}$.

The expected compound (1) was obtained in 28 to $54 \%$ yield. Adding a catalyst to the reaction was always detrimental to the yield when compared to a catalyst-free reaction performed with the same stoichiometry and temperature. The best result was obtained without catalyst using one equivalent of benzaldehyde and two equivalents of pyrrolidine and malonitrile (Table 1, Entry 2). For the synthesis of compound 1, this solvent substitution proved to be advantageous over the above-mentioned studies that used chloroform (32\%) [23] and ethanol (52\%) [24]. However, our yield was lower when compared to that of the team that performed the reaction in methanol (79\%) [25]. Nevertheless, it should be highlighted that the $79 \%$ yield was obtained with the addition of DMAP ( $20 \mathrm{~mol} \%$ ) as catalyst. To test which parameter influenced the yield, we performed the reaction in methanol without catalyst and then verified that the use of eucalyptol resulted in a higher yield when the reaction mixture was catalyst-free. With the best reaction conditions in hand, we proceeded to analyze the scope and limitations of the reaction.

The derivatives (1-6) were synthesized in 45 to $68 \%$ yield (Figure 3 ). The nature of the aldehyde did not cause major disparities in the yield of the different final compounds. Then, considering the aldehyde that presented the highest yield, the potential of eucalyptol using other sources of amines (piperidine, thiomorpholine, 2,6-dimethylmorpholine, and 1phenylpiperazine) was evaluated: the final compounds $\mathbf{7 - 1 0}$ were synthesized in moderate to good yields (Figure 4). 


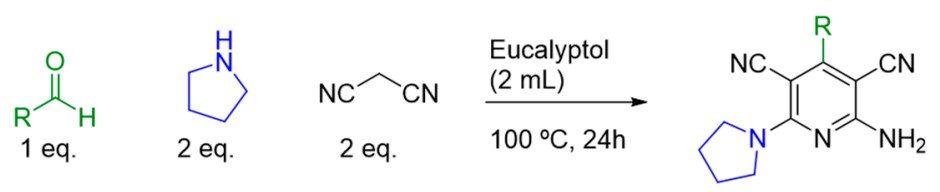<smiles>N#Cc1c(N)nc(N2CCCC2)c(C#N)c1-c1ccccc1</smiles>

1, $54 \%$<smiles>N#Cc1c(N)nc(N2CCCC2)c(C#N)c1-c1ccc(F)cc1</smiles>

4, $64 \%$

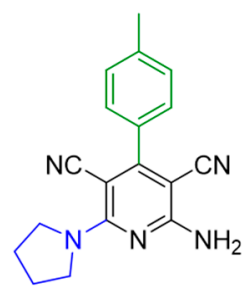

2, $68 \%$<smiles>COc1ccc(-c2c(C#N)c(N)nc(N3CCCC3)c2C#N)cc1</smiles>

5, $51 \%$

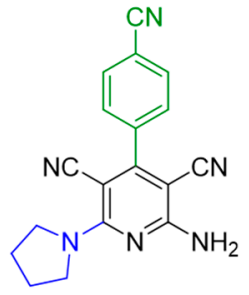

$3,45 \%$

Figure 3. Scope and limitations of multicomponent reaction for the synthesis of highly functionalized pyridines.

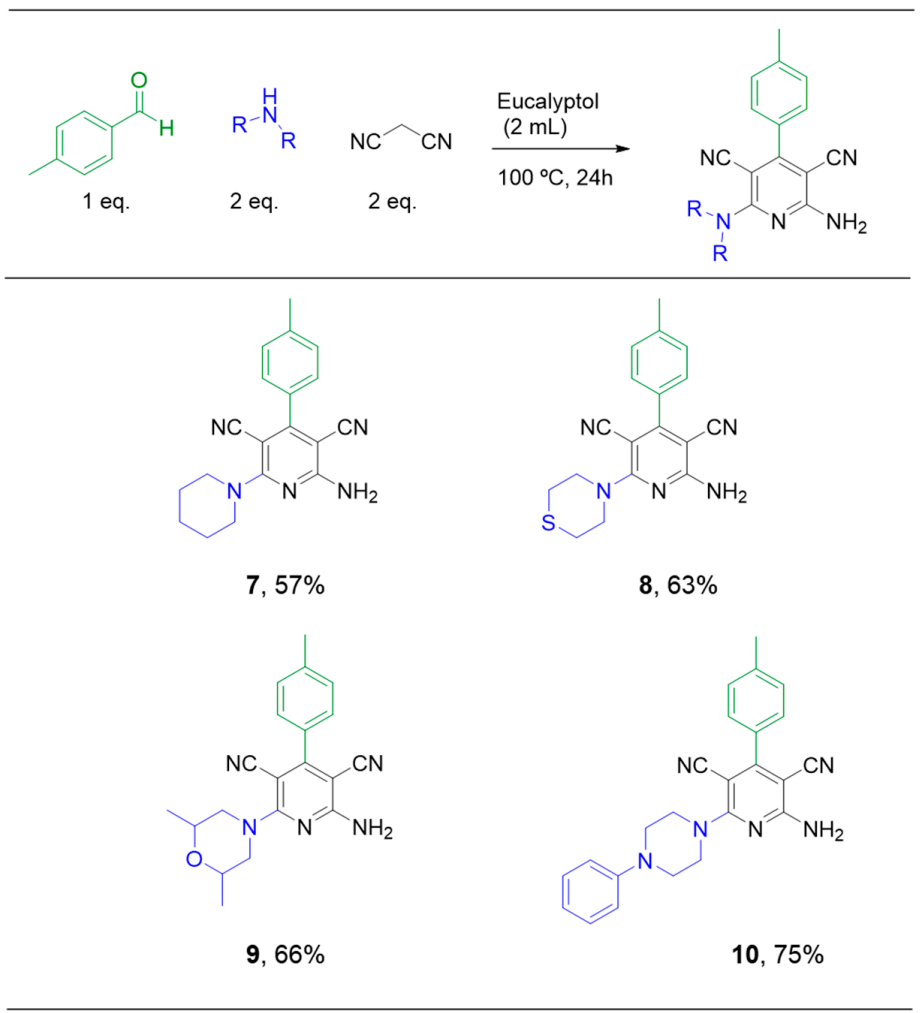

Figure 4. Multicomponent reaction for the synthesis of highly functionalized pyridines. 


\subsection{Palladium Catalyzed Cyanation}

The second reaction explored with eucalyptol as solvent was palladium-catalyzed cyanation. This reaction offers an appropriate alternative to the Rosenmund-Von Braun reaction [26-30], which frequently employs severe reaction conditions and sometimes needs an intensive work up. Due to all of these features and properties, efforts were made to find greener conditions. For this study, we used three compounds commonly used in our team to build molecules of interest with biological potential [15,31,32]. Each compound underwent an optimization study in order to find the ideal conditions. To the best of our knowledge, here, we present the first cyanation process of these scaffolds. After reviewing previously reported information [33-39] related to the cyanation of $O, S, N$-containing heterocycles, we performed the optimization on 4-chlorothieno[3,2-d]pyrimidine, 7-chlorothieno[3,2-b]pyridine, and 7-bromo-6-phenyl-thieno[2,3-b]pyrazine.

In the literature, $\mathrm{Zn}(\mathrm{CN})_{2}$ is often used as cyanide source. The reaction can occur because, as the cyanide nucleophile is a strong $\sigma$-donor and can be fatal to the catalytic system, it is essential to keep its concentration low in the reaction. An unfavorable point is its limited solubility in DMF $\left(1.8 \times 10^{-4} \mathrm{~g} / \mathrm{mL}\right.$ at $\left.80^{\circ} \mathrm{C}\right)$, which is a solvent commonly used in these reactions [40].

Another source of cyanide (non-toxic), $\mathrm{K}_{4}\left[\mathrm{Fe}(\mathrm{CN})_{6}\right]$, has also been described and can be used in a mixture with palladium catalysts to obtain aryl nitriles from their corresponding halides [41]. From this background, we tested reaction conditions using eucalyptol as solvent. Compound 11 was obtained in a yield from 7 to $56 \%$ (Table 2).

Table 2. Optimization of cyanation.

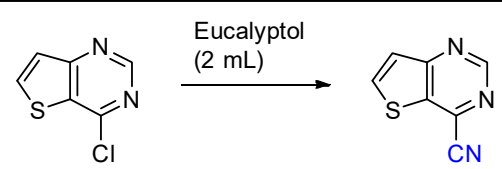

11

\begin{tabular}{|c|c|c|c|c|c|c|}
\hline Entry & Pd (eq.) & Lig (eq.) & CN (eq.) & $\mathrm{T}\left({ }^{\circ} \mathrm{C}\right)$ & $t(h)$ & Yield a $(\%)$ \\
\hline 1 & $\mathrm{Pd}\left(\mathrm{PPh}_{3}\right)_{4}(0.07)$ & - & $\mathrm{Zn}(\mathrm{CN})_{2}(0.6)$ & 100 & 96 & 7 \\
\hline $2^{c}$ & $\mathrm{Pd}_{2}(\mathrm{dba})_{3}(0.05)$ & dppf (0.05) & $\mathrm{Zn}(\mathrm{CN})_{2}(0.6)$ & 100 & 96 & 11 \\
\hline $3^{c}$ & $\mathrm{Pd}_{2}(\mathrm{dba})_{3}(0.05)$ & dppf (0.05) & $\mathrm{Zn}(\mathrm{CN})_{2}(0.6)$ & 140 & 44 & 55 \\
\hline 4 & $\mathrm{Pd}\left(\mathrm{PPh}_{3}\right)_{4}(0.05)$ & - & $\mathrm{KCN}(1.5)$ & 140 & 61 & 0 \\
\hline 5 & $\mathrm{PdCl}_{2}\left(\mathrm{PPh}_{3}\right)_{2}(0.05)$ & - & $\mathrm{KCN}(2)$ & 140 & 61 & 0 \\
\hline $6^{d}$ & $\mathrm{Pd}(\mathrm{OAc})_{2}(0.05)$ & dppe (0.1) & $\mathrm{KCN}(1)$ & 140 & 61 & 0 \\
\hline $7^{\mathrm{d}}$ & $\mathrm{Pd}(\mathrm{OAc})_{2}(0.05)$ & dppe (0.1) & $\mathrm{KCN}(1)$ & 140 & 61 & 0 \\
\hline $8^{d}$ & $\mathrm{Pd}(\mathrm{OAc})_{2}(0.05)$ & dppe (0.1) & $\mathrm{KCN}(1)$ & 140 & 61 & 0 \\
\hline $9^{d}$ & $\mathrm{Pd}(\mathrm{OAc})_{2}(0.05)$ & dppe (0.1) & $\mathrm{KCN}(1)$ & 140 & 61 & 0 \\
\hline 10 & $\mathrm{Pd}_{2}(\mathrm{dba})_{3}(0.1)$ & dppf (0.4) & $\mathrm{KCN}(2)$ & 140 & 44 & 24 \\
\hline $11^{\mathrm{e}}$ & $\mathrm{Pd}(\mathrm{OAc})_{2}(0.03)$ & cataCXium (0.09) & $\mathrm{K}_{4}\left[\mathrm{Fe}(\mathrm{CN})_{6}\right]^{\mathrm{b}}(0.2)$ & 140 & 41 & traces \\
\hline 12 & $\mathrm{Pd}_{2}(\mathrm{dba})_{3}(0.03)$ & cataCXium (0.09) & $\mathrm{K}_{4}\left[\mathrm{Fe}(\mathrm{CN})_{6}\right]^{\mathrm{b}}(0.2)$ & 140 & 41 & traces \\
\hline $13^{\mathrm{e}}$ & $\mathrm{Pd}(\mathrm{TFA})_{2}(0.03)$ & TTBP. $\mathrm{HBF}_{4}(0.09)$ & $\mathrm{K}_{4}\left[\mathrm{Fe}(\mathrm{CN})_{6}\right]^{\mathrm{b}}(0.2)$ & 140 & 41 & traces \\
\hline $14^{\mathrm{e}}$ & $\mathrm{PdCl}_{2}(0.03)$ & $\mathrm{TTBP} \cdot \mathrm{HBF}_{4}(0.09)$ & $\mathrm{K}_{4}\left[\mathrm{Fe}(\mathrm{CN})_{6}\right]^{\mathrm{b}}(0.2)$ & 140 & 41 & traces \\
\hline $15^{c}$ & $\mathrm{Pd}_{2}(\mathrm{dba})_{3}(0.05)$ & $\operatorname{dppf}(0.05)$ & $\mathrm{Zn}(\mathrm{CN})_{2}(0.6)$ & 140 & 96 & 43 \\
\hline $16^{\mathrm{e}}$ & $\mathrm{Pd}(\mathrm{OAc})_{2}(0.05)$ & X-Phos (0.1) & $\begin{array}{c}\mathrm{K}_{4}\left[\mathrm{Fe}(\mathrm{CN})_{6}\right]^{\mathrm{b}} \\
(0.25)\end{array}$ & 140 & 60 & 56 \\
\hline $17^{\mathrm{c}}$ & $\mathrm{Pd}_{2}(\mathrm{dba})_{3}(0.05)$ & $\mathrm{PCy}_{3}(0.05)$ & $\mathrm{Zn}(\mathrm{CN})_{2}(0.6)$ & 140 & 48 & 48 \\
\hline $18^{\mathrm{e}}$ & $\mathrm{Pd}(\mathrm{OAc})_{2}(0.05)$ & dppf (0.1) & $\mathrm{K}_{4}\left[\mathrm{Fe}(\mathrm{CN})_{6}\right]^{\mathrm{b}}(0.2)$ & 140 & 60 & 43 \\
\hline $19^{c}$ & $\mathrm{Pd}_{2}(\mathrm{dba})_{3}(0.05)$ & dppf (0.1) & $\mathrm{Zn}(\mathrm{CN})_{2}(0.6)$ & 170 & 26 & 39 \\
\hline 20 & - & - & $\mathrm{NaCN}(5)$ & $\mathrm{rt}$ & 26 & 0 \\
\hline 21 & - & - & $\mathrm{NaCN}(5)$ & 170 & 24 & 0 \\
\hline
\end{tabular}

${ }^{a}$ isolated yield after purification via flash chromatography. ${ }^{\mathrm{b}} \mathrm{K}_{4}\left[\mathrm{Fe}(\mathrm{CN})_{6}\right] \cdot 3 \mathrm{H}_{2} \mathrm{O} .{ }^{\mathrm{c}} 0.2$ equivalent of $\mathrm{Zn}$ was added. ${ }^{\mathrm{d}} 0.2$ equivalent of amine co-catalyst was added. Entry 6: TMEDA; Entry 7: Sparteine; Entry 8: 2,2-bipyridine; Entry 9: 1-adamantylamine. ${ }^{\mathrm{e}} 0.2$ equivalent of base was added. Entry 11, 13, 14 and 18: $\mathrm{Na}_{2} \mathrm{CO}_{3} ;$ Entry 16: $\mathrm{K}_{2} \mathrm{CO}_{3}$. 
Starting from 7-chlorothieno[3,2-b]pyridine and based on our results with 4chlorothieno[3,2-d]pyrimidine (Table 2), we looked for the conditions that would result in the highest yield. The best outcome was achieved when the reaction was performed using eucalyptol as solvent with $\mathrm{Pd}_{2}(\mathrm{dba})_{3}(5 \mathrm{~mol} \%)$, $\mathrm{dppf}(10 \mathrm{~mol} \%), \mathrm{Zn}(\mathrm{CN})_{2}$ $(60 \mathrm{~mol} \%), \mathrm{Zn}(20 \mathrm{~mol} \%)$ at $170{ }^{\circ} \mathrm{C}$ for $26 \mathrm{~h}$ (Table 3$)$.

Table 3. Optimization of cyanation.

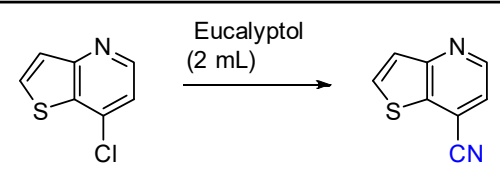

12

\begin{tabular}{ccccccc}
\hline Entry & Pd (eq.) & Lig (eq.) & $\mathbf{C N}$ (eq.) & T ( $\left.{ }^{\circ} \mathbf{C}\right)$ & t (h) & Yield ${ }^{\text {a }}(\%)$ \\
\hline $1^{\mathrm{c}}$ & $\mathrm{Pd}_{2}(\mathrm{dba})_{3}(0.05)$ & $\mathrm{PCy}_{3}(0.1)$ & $\mathrm{Zn}(\mathrm{CN})_{2}(0.06)$ & 170 & 48 & 9 \\
$2^{\mathrm{c}, \mathrm{d}}$ & $\left.\mathrm{Pd}_{(\mathrm{OAc}}\right)_{2}(0.05)$ & $\mathrm{X}-\mathrm{Phos}(0.1)$ & $\mathrm{K}_{4}\left[\mathrm{Fe}(\mathrm{CN})_{6}\right]^{\mathrm{b}}(0.2)$ & 170 & 48 & 0 \\
$3^{\mathrm{c}}$ & $\mathrm{Pd}_{2}(\mathrm{dba})_{3}(0.05)$ & $\mathrm{dppf}(0.1)$ & $\mathrm{Zn}(\mathrm{CN})_{2}(0.6)$ & 170 & 26 & 61 \\
\hline
\end{tabular}

${ }^{a}$ isolated yield after purification via flash chromatography. ${ }^{b} \mathrm{~K}_{4}\left[\mathrm{Fe}(\mathrm{CN})_{6}\right] \cdot 3 \mathrm{H}_{2} \mathrm{O} .{ }^{c} 0.2$ equivalent of $\mathrm{Zn}$ was added. ${ }^{\mathrm{d}} 0.2$ equivalent of $\mathrm{K}_{2} \mathrm{CO}_{3}$ was added.

To test the versatility of conditions over bromine derivatives, we chose a molecule synthesized in a previous study reported by our team [31] (Figure 5).

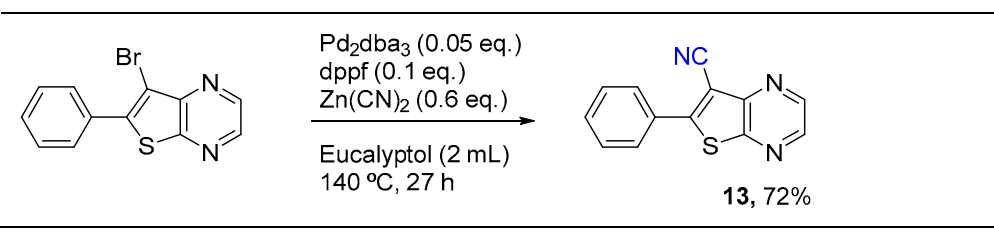

Figure 5. Palladium catalyzed cyanation of 7-bromo-6-phenyl-thieno[2,3-b]pyrazine.

The desired product $\mathbf{1 3}$ was obtained in good yield using eucalyptol as solvent at $140{ }^{\circ} \mathrm{C}$ for $27 \mathrm{~h}$ with $\mathrm{Pd}_{2}(\mathrm{dba})_{3} / \mathrm{dppf}$ as catalytic system and $\mathrm{Zn}(\mathrm{CN})_{2}$ as cyanide source.

\subsection{Hiyama Coupling}

Hiyama coupling is a palladium-catalyzed C-C bond formation between aryl, alkenyl, or alkyl halides or pseudohalides and organosilanes. Its particularity lies in the requirement for a fluoride ion or a base as activating agent $[42,43]$. This coupling was chosen in order to compare it with the results obtained and reported previously by our team [15] on the performance of Sonogashira coupling using eucalyptol as solvent on $O, S, N$-heterocycles.

As with the previous two reactions reported above, this work started with a literature review [43-47] to test the conditions for our scaffold and identify the best coupling conditions.

Optimization was achieved starting from 7-chlorothieno[3,2-b]pyridine and 1-phenyl2-trimethylsilylacetylene and by varying the amount and type of Pd source with or without ligand as well as the type and amount of activating agent (fluoride ion or a base). Reactions with eucalyptol were conducted at $100{ }^{\circ} \mathrm{C}$ for durations summarized in Table 4 . 
Table 4. Optimization of Hiyama coupling.

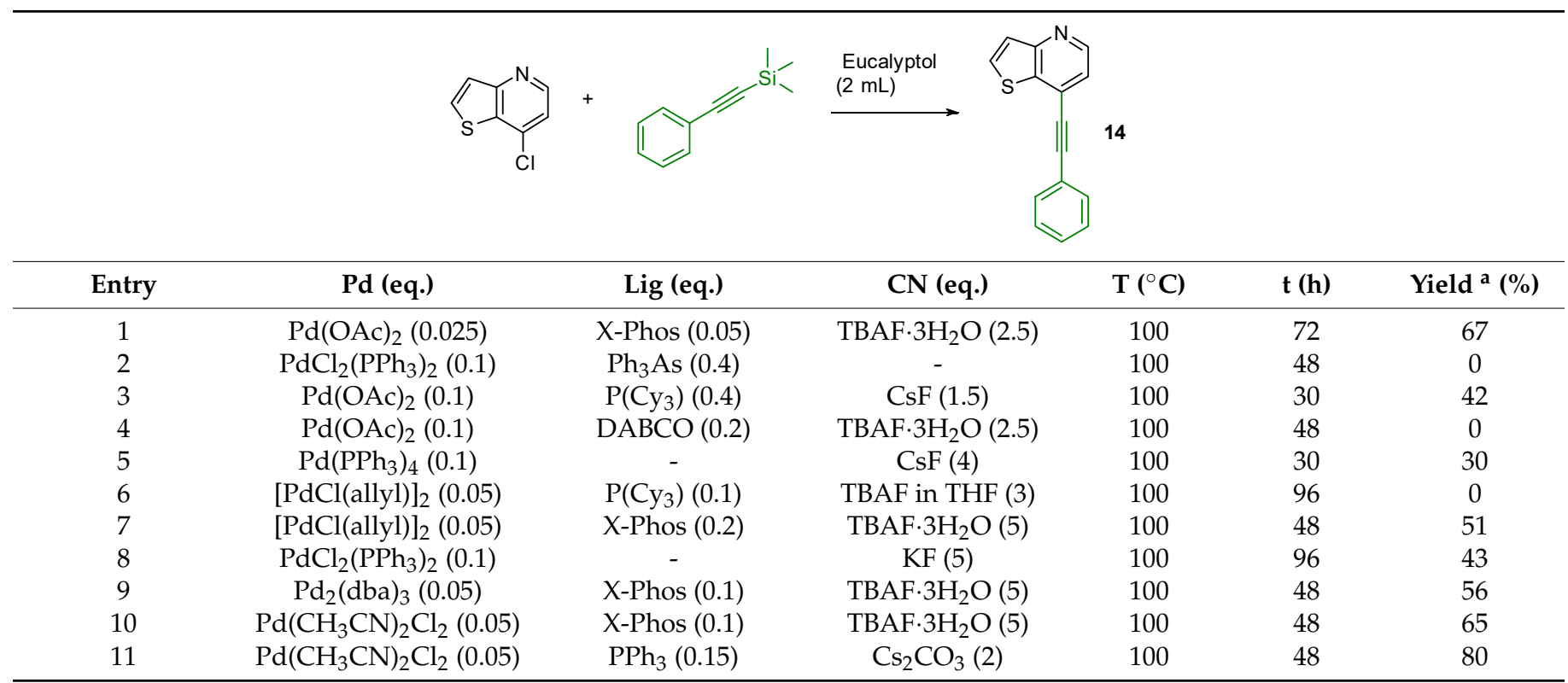

${ }^{a}$ isolated yield after purification via flash chromatography.

Process optimization led to the isolation of compound 14 in a yield ranging from 30 to $80 \%$. The best reaction conditions using eucalyptol as solvent were achieved at $100{ }^{\circ} \mathrm{C}$ for $48 \mathrm{~h}$ with $\mathrm{Pd}\left(\mathrm{CH}_{3} \mathrm{CN}\right)_{2} \mathrm{Cl}_{2} / \mathrm{PPh}_{3}$ as a catalytic system and $\mathrm{Cs}_{2} \mathrm{CO}_{3}$ as a base.

Based on our results (Table 4, Entry 11), the scope and limitations of the Hiyama coupling on 7-chlorothieno[3,2-b]pyridine were assessed using several silylacetylenes (Table 4).

Compounds 14-19 substituted in position 7 were synthesized in moderate to good yield, demonstrating the generalizability of this method using eucalyptol as solvent (Figure 6).

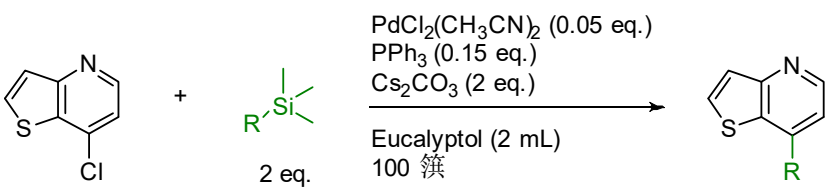

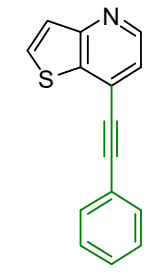<smiles>C=CCc1ccnc2ccsc12</smiles><smiles>FC(F)(F)c1ccc(C#Cc2ccnc3ccsc23)cc1</smiles>

$14,80 \%(48 \mathrm{~h}) \quad 15,53 \%(40 \mathrm{~h})$<smiles>C(#Cc1ccnc2ccsc12)c1ccoc1</smiles><smiles>COc1ccc(C#Cc2ccnc3ccsc23)cc1</smiles>

$18,81 \%(48 h) \quad 19,51 \%(36 h)$

Figure 6. Scope and limitations of the Hiyama coupling from 7-chlorothieno[3,2-b]pyridine.

From these results, we explored the same conditions on 4-chlorofuro[3,2-c]pyridine.

Two examples (20-21) were synthesized in low yield by Hiyama coupling (Figure 7). This scaffold showed lower reactivity. This aspect had already been observed when we synthesized the same products by Sonogashira coupling using eucalyptol [15]. 


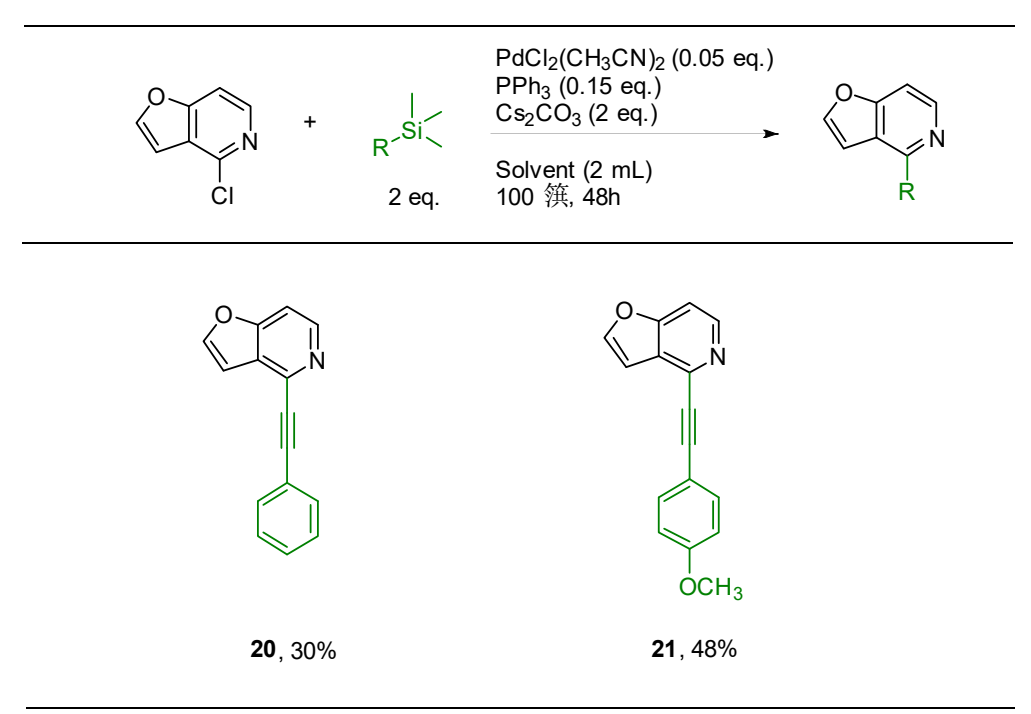

Figure 7. Scope and limitations of the Hiyama coupling from 4-chlorofuro[3,2-c]pyridine.

\subsection{Recyclability of the Solvent}

As the reusability of the solvent is essential from an economic and environmental perspective, we have already shown its feasibility in Pd-mediated cross-coupling reactions in our previous work [15-17], wherein an average 70\% solvent recovery (using a rotary evaporator system) was observed for each reaction series without noticeable loss of properties. Although the boiling point of eucalyptol is relatively high, it is possible to evaporate it, in a few minutes, with a normal pump and recirculating chiller in a classical rotary evaporator system.

\section{Materials and Methods}

\subsection{General Methods}

All reagents used herein were purchased from commercial suppliers Sigma Aldrich, St Quentin Fallavier Cedex, France; Fluorochem, Derbyshire, SK131QH, UK. All the reactions were monitored by thin-layer chromatography (TLC) using silica gel (60 F254) plates. Flash column chromatographies were performed on silica gel 60 (230-400 mesh, 0.040-0.063 mm). ${ }^{1} \mathrm{H}$ and ${ }^{13} \mathrm{C}$ NMR spectra were recorded on a Bruker avance II spectrometer (Bruker, Wissembourg, France) at $250 \mathrm{MHz}(13 \mathrm{C}, 62.9 \mathrm{MHz})$ and on a Bruker avance III HD nanobay (Bruker, Wissembourg, France) $400 \mathrm{MHz}$ (13C $100.62 \mathrm{MHz}$ ). The following abbreviations: b: broad, s: singlet, d: doublet, t: triplet, q: quartet, p: pentuplet, m: multiplet are used for the proton spectra multiplicities. Coupling constants (J) are reported in Hertz $(\mathrm{Hz})$. Multiplicities were determined by the DEPT 135 sequence and chemical shifts are given from tetramethylsilane (TMS) or deuterated solvent (MeOH-d4, Chloroform-d) as internal standard. High-resolution mass spectra (HRMS) were carried out on a Maxis UHR-q-TOF mass spectrometer (Bruker, Wissembourg, France) Bruker 4G in electrospray ionization (ESI) mode (Bruker, Wissembourg, France). Melting points $\left(\mathrm{mp}\left[{ }^{\circ} \mathrm{C}\right]\right)$ were determined in open capillary tubes and are uncorrected.

\subsection{Multicomponent Reaction: General Procedure for Synthesis of Compounds 1-10}

A mixture of aldehyde (50 mg; 1 eq.), amino derivative (2 eq.), malonitrile ( 2 eq.) in Eucalyptol $(2 \mathrm{~mL})$ was stirred at $100^{\circ} \mathrm{C}$ for $24 \mathrm{~h}$. The reaction was followed by TLC. After completion, the reaction was cooled to room temperature and the mixture was concentrated under vacuum. The solid obtained was purified by flash chromatography using a mixture of AcOEt/petroleum ether.

2-amino-4-phenyl-6-(pyrrolidin-1-yl)pyridine-3,5-dicarbonitrile (1). Yellow solid (74 mg, $54 \%){ }^{1} \mathrm{H} \mathrm{NMR}\left(400 \mathrm{MHz}, \mathrm{CDCl}_{3}\right) \delta 1.97(\mathrm{t}, J=6.7 \mathrm{~Hz}, 4 \mathrm{H}), 3.81(\mathrm{~s}, 4 \mathrm{H}), 5.31$ (d, $J=17.5 \mathrm{~Hz}$, 2H), $7.49(\mathrm{dtt}, J=10.0,6.3,2.9 \mathrm{~Hz}, 5 \mathrm{H}) \mathrm{ppm} .{ }^{13} \mathrm{C} \mathrm{NMR}\left(101 \mathrm{MHz}, \mathrm{CDCl}_{3}\right) \delta 25.4(2 \times \mathrm{CH})$, 
$49.6(2 \times C H), 81.1(\mathrm{C}), 82.0(\mathrm{C}), 116.8(\mathrm{C}), 118.2(\mathrm{C}), 128.5(2 \times \mathrm{CH}), 128.7(2 \times \mathrm{CH}), 130.2(\mathrm{CH})$, 135.0 (C), 157.5 (C), 159.3 (C), 162.1 (C) ppm. [CAS: 77034-27-6].

2-amino-6-(pyrrolidin-1-yl)-4-(p-tolyl)pyridine-3,5-dicarbonitrile (2). Yellow solid (86 mg, $68 \%)$, m.p. $234-236{ }^{\circ} \mathrm{C} .{ }^{1} \mathrm{H} \mathrm{NMR}\left(400 \mathrm{MHz}, \mathrm{CDCl}_{3}\right) \delta 1.97(\mathrm{t}, J=6.4 \mathrm{~Hz}, 4 \mathrm{H}), 2.41(\mathrm{~s}, 3 \mathrm{H})$, $3.80(\mathrm{~s}, 4 \mathrm{H}), 5.31(\mathrm{~d}, J=11.2 \mathrm{~Hz}, 2 \mathrm{H}), 7.30(\mathrm{~d}, J=7.9 \mathrm{~Hz}, 2 \mathrm{H}), 7.37(\mathrm{~d}, J=8.0 \mathrm{~Hz}, 2 \mathrm{H}) \mathrm{ppm}$. ${ }^{13} \mathrm{C}$ NMR $\left(101 \mathrm{MHz}, \mathrm{CDCl}_{3}\right) \delta 21.5(\mathrm{CH}), 25.4(2 \times \mathrm{CH}), 49.6(2 \times \mathrm{CH}), 81.0(\mathrm{C}), 82.0(\mathrm{C}), 117.0$ (C), $118.4(\mathrm{C}), 128.4(2 \times C H), 129.4(2 \times C H), 132.0(\mathrm{C}), 140.4(\mathrm{C}), 157.6(\mathrm{C}), 159.4(\mathrm{C}), 162.2(\mathrm{C})$ ppm. HRMS: calcd for $\mathrm{C}_{18} \mathrm{H}_{18} \mathrm{~N}_{5} \mathrm{~S}[\mathrm{M}+\mathrm{H}]^{+} 336.1277$, found 336.1280 .

2-amino-4-(4-cyanophenyl)-6-(pyrrolidin-1-yl)pyridine-3,5-dicarbonitrile (3). Yellow solid (54 mg, 45\%), m.p. $258-260{ }^{\circ} \mathrm{C} .{ }^{1} \mathrm{H}$ NMR $\left(400 \mathrm{MHz}, \mathrm{CDCl}_{3}\right) \delta 1.99(\mathrm{~s}, 4 \mathrm{H}), 3.81(\mathrm{~s}, 4 \mathrm{H})$, $5.37(\mathrm{~s}, 2 \mathrm{H}), 7.58(\mathrm{~d}, J=8.1 \mathrm{~Hz}, 2 \mathrm{H}), 7.81(\mathrm{~d}, J=8.1 \mathrm{~Hz}, 2 \mathrm{H}) \mathrm{ppm} .{ }^{13} \mathrm{C} \mathrm{NMR}(101 \mathrm{MHz}$, $\left.\mathrm{CDCl}_{3}\right) \delta 25.3(2 \times \mathrm{CH}), 49.6(2 \times \mathrm{CH}), 80.6(\mathrm{C}), 81.6(\mathrm{C}), 114.2(\mathrm{C}), 116.1(\mathrm{C}), 117.7(\mathrm{C}), 118.0$ (C), $129.4(2 \times C H), 132.6(2 \times C H), 139.4(\mathrm{C}), 157.1(\mathrm{C}), 159.1$ (C), 159.9 (C) ppm. HRMS: calcd for $\mathrm{C}_{18} \mathrm{H}_{15} \mathrm{~N}_{6}[\mathrm{M}+\mathrm{H}]^{+}$315.1353, found 315.1352.

2-amino-4-(4-fluorophenyl)-6-(pyrrolidin-1-yl)pyridine-3,5-dicarbonitrile (4). Yellow solid (79 mg, 64\%), m.p. $253-255^{\circ} \mathrm{C} .{ }^{1} \mathrm{H}$ NMR $\left(400 \mathrm{MHz}, \mathrm{CDCl}_{3}\right) \delta 1.98(\mathrm{t}, J=6.4 \mathrm{~Hz}, 4 \mathrm{H}), 3.80$ $(\mathrm{s}, 4 \mathrm{H}), 5.35(\mathrm{~s}, 2 \mathrm{H}), 7.19(\mathrm{t}, J=8.6 \mathrm{~Hz}, 2 \mathrm{H}), 7.47(\mathrm{dd}, J=8.4,5.3 \mathrm{~Hz}, 2 \mathrm{H}), \mathrm{ppm} .{ }^{13} \mathrm{C}$ NMR $\left(101 \mathrm{MHz}, \mathrm{CDCl}_{3}\right) \delta 25.4(2 \times \mathrm{CH}), 49.6(2 \times \mathrm{CH}), 81.5(\mathrm{~d}, \mathrm{~J}=94 \mathrm{~Hz}, \mathrm{C}), 115.9(\mathrm{CH}), 116.7$ (C), $116.1(\mathrm{CH}), 118.1(\mathrm{C}), 130.6(\mathrm{CH}), 130.7(\mathrm{CH}), 131.0(\mathrm{C}), 157.5(\mathrm{C}), 159.3(\mathrm{C}), 161.0(\mathrm{C})$, $163.8(\mathrm{~d}, J=250 \mathrm{~Hz}, \mathrm{C}), \mathrm{ppm} .{ }^{19} \mathrm{~F}$ NMR $\left(376 \mathrm{MHz}, \mathrm{CDCl}_{3}\right) \delta-110.05 \mathrm{ppm}$. HRMS: calcd for $\mathrm{C}_{17} \mathrm{H}_{15} \mathrm{FN}_{5}[\mathrm{M}+\mathrm{H}]^{+}$308.1306, found 308.1309.

2-amino-4-(4-methoxyphenyl)-6-(pyrrolidin-1-yl)pyridine-3,5-dicarbonitrile (5). Yellow solid $(60 \mathrm{mg}, 51 \%){ }^{1} \mathrm{H}$ NMR $\left(400 \mathrm{MHz}, \mathrm{CDCl}_{3}\right) \delta 7.44(\mathrm{~d}, J=8.4 \mathrm{~Hz}, 2 \mathrm{H}), 7.01(\mathrm{~d}, J=8.5 \mathrm{~Hz}, 2 \mathrm{H})$, $5.31(\mathrm{~s}, 2 \mathrm{H}), 3.83(\mathrm{~d}, J=21.4 \mathrm{~Hz}, 7 \mathrm{H}), 1.97(\mathrm{t}, J=6.4 \mathrm{~Hz}, 4 \mathrm{H}) \mathrm{ppm} .{ }^{13} \mathrm{C}$ NMR $(101 \mathrm{MHz}$, $\left.\mathrm{CDCl}_{3}\right) \delta 25.4(2 \times \mathrm{CH}), 49.6(2 \times \mathrm{CH}), 55.3(\mathrm{CH}), 81.0(\mathrm{C}), 81.9(\mathrm{C}), 114.1(2 \times \mathrm{CH}), 117.1(\mathrm{C})$, $118.5(\mathrm{C}), 127.0(\mathrm{C}), 130.2(2 \times C H), 157.8(\mathrm{C}), 159.43$ (C), 161.1 (C), 161.8 (C) ppm. [CAS: 77034-28-7].

2-amino-4-cyclohexyl-6-(pyrrolidin-1-yl)pyridine-3,5-dicarbonitrile (6). Yellow solid (71 mg,

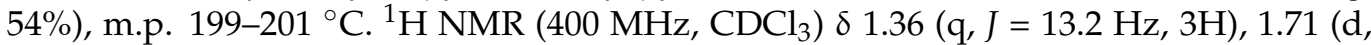
$J=12.2 \mathrm{~Hz}, 3 \mathrm{H}), 1.95-1.85(\mathrm{~m}, 5 \mathrm{H}), 2.10(\mathrm{q}, J=12.2 \mathrm{~Hz}, 2 \mathrm{H}), 3.07(\mathrm{t}, J=12.4 \mathrm{~Hz}, 1 \mathrm{H}), 3.73(\mathrm{~s}$, 4H), 5.29 (s, 2H) ppm. $\left.{ }^{13} \mathrm{C} \mathrm{NMR} \mathrm{(101} \mathrm{MHz,} \mathrm{CDCl}_{3}\right) \delta 167.4(\mathrm{C}), 160.2(\mathrm{C}), 158.2(\mathrm{C}), 118.2$ $(\mathrm{C}), 117.6(\mathrm{C}), 81.5(\mathrm{C}), 79.2(\mathrm{C}), 60.4(\mathrm{C}), 49.6(2 \times \mathrm{CH}), 44.3(\mathrm{CH}), 29.7(2 \times \mathrm{CH}), 26.5(2 \times \mathrm{CH})$, $25.4(\mathrm{CH}), 25.3(\mathrm{CH})$ ppm. HRMS: calcd for $\mathrm{C}_{17} \mathrm{H}_{22} \mathrm{~N}_{5}[\mathrm{M}+\mathrm{H}]^{+} 296.1870$, found 296.1871 .

2-amino-6-(piperidin-1-yl)-4-(p-tolyl)pyridine-3,5-dicarbonitrile (7). Yellow solid (75 mg, $57 \%){ }^{1} \mathrm{H}$ NMR $\left(400 \mathrm{MHz}, \mathrm{CDCl}_{3}\right) \delta 7.39(\mathrm{~d}, J=8.1 \mathrm{~Hz}, 2 \mathrm{H}), 7.30(\mathrm{~d}, J=7.9 \mathrm{~Hz}, 2 \mathrm{H}), 5.36$ (s, 2H), $3.79(\mathrm{~s}, 4 \mathrm{H}), 2.41(\mathrm{~s}, 3 \mathrm{H}), 1.70(\mathrm{~s}, 6 \mathrm{H}) \mathrm{ppm} .{ }^{13} \mathrm{C} \mathrm{NMR}\left(101 \mathrm{MHz}, \mathrm{CDCl}_{3}\right) \delta 162.4$ (C), $161.3(\mathrm{C}), 159.5(\mathrm{C}), 140.7(\mathrm{C}), 131.9(\mathrm{C}), 129.5(\mathrm{CH}), 129.0(\mathrm{CH}), 128.6(\mathrm{CH}), 117.9(\mathrm{C})$, $116.7(\mathrm{C}), 83.6(\mathrm{C}), 81.6(\mathrm{C}), 49.2(2 \times \mathrm{CH}), 26.0(2 \times \mathrm{CH}), 24.4(2 \times \mathrm{CH}), 21.5(\mathrm{CH}) \mathrm{ppm}$. [CAS: 1268160-67-3].

2-amino-6-thiomorpholino-4-( -tolyl)pyridine-3,5-dicarbonitrile (8). Yellow solid (88 $\mathrm{mg}$, $63 \%)$, m.p. $223-225^{\circ} \mathrm{C} .{ }^{1} \mathrm{H}$ NMR $\left(400 \mathrm{MHz}, \mathrm{CDCl}_{3}\right) \delta 7.38(\mathrm{~d}, J=8.2 \mathrm{~Hz}, 2 \mathrm{H}), 7.31(\mathrm{~d}$, $J=8.0 \mathrm{~Hz}, 2 \mathrm{H}), 5.46(\mathrm{~s}, 2 \mathrm{H}), 4.11-4.06(\mathrm{~m}, 4 \mathrm{H}), 2.76(\mathrm{dd}, J=7.4,2.8 \mathrm{~Hz}, 4 \mathrm{H}), 2.41(\mathrm{~s}, 3 \mathrm{H})$ ppm. ${ }^{13} \mathrm{C}$ NMR (101 MHz, CDCl $) \delta 162.5$ (C), 161.7 (C), 159.5 (C), 140.9 (C), 131.6 (C), 129.6 $(2 \times \mathrm{CH}), 128.6(2 \times \mathrm{CH}), 117.6(\mathrm{C}), 116.3(\mathrm{C}), 84.2(\mathrm{C}), 82.6(\mathrm{C}), 50.8(2 \times \mathrm{CH}), 27.4(2 \times \mathrm{CH}), 21.5$ (CH) ppm. HRMS: calcd for $\mathrm{C}_{18} \mathrm{H}_{18} \mathrm{~N}_{5} \mathrm{~S}[\mathrm{M}+\mathrm{H}]^{+} 336.1277$, found 336.1280.

2-amino-6-(2,6-dimethylmorpholino)-4-(p-tolyl)pyridine-3,5-dicarbonitrile (9). Yellow solid (95 mg, 66\%), m.p. $238-240{ }^{\circ} \mathrm{C} .{ }^{1} \mathrm{H}$ NMR $\left(400 \mathrm{MHz}, \mathrm{CDCl}_{3}\right) \delta 7.39$ (d, J = 8.2 Hz, 2H), 7.31 $(\mathrm{d}, J=7.9 \mathrm{~Hz}, 2 \mathrm{H}), 5.46(\mathrm{~s}, 2 \mathrm{H}), 4.41(\mathrm{~d}, J=13.3 \mathrm{~Hz}, 2 \mathrm{H}), 2.78(\mathrm{dd}, J=13.3,10.5 \mathrm{~Hz}, 2 \mathrm{H})$, $2.41(\mathrm{~s}, 3 \mathrm{H}), 1.23(\mathrm{~d}, J=6.3 \mathrm{~Hz}, 6 \mathrm{H}) \mathrm{ppm} .{ }^{13} \mathrm{C} \mathrm{NMR}\left(101 \mathrm{MHz}, \mathrm{CDCl}_{3}\right) \delta 162.4(\mathrm{C}), 161.1(\mathrm{C})$, $159.5(2 \times C), 140.9(2 \times C), 131.6(\mathrm{C}), 129.5(2 \times C H), 128.6(2 \times C H), 117.7(\mathrm{C}), 116.4(\mathrm{C}), 83.9(\mathrm{C})$, $82.4(\mathrm{C}), 71.7(\mathrm{CH}), 53.2(\mathrm{CH}), 21.5(\mathrm{CH}), 18.7(2 \times \mathrm{CH})$ ppm. HRMS: calcd for $\mathrm{C}_{20} \mathrm{H}_{22} \mathrm{~N}_{5} \mathrm{O}$ $[\mathrm{M}+\mathrm{H}]^{+} 348.1819$, found 348.1818 .

2-amino-6-(4-phenylpiperazin-1-yl)-4-(p-tolyl)pyridine-3,5-dicarbonitrile (10). Yellow solid (109 mg, 75\%), m.p. $220-222{ }^{\circ} \mathrm{C} .{ }^{1} \mathrm{H}$ NMR $\left(400 \mathrm{MHz}, \mathrm{CDCl}_{3}\right) \delta 7.41(\mathrm{~d}, J=8.2 \mathrm{~Hz}, 2 \mathrm{H})$, 
7.35-7.28 (m, 4H), 6.97-6.89 (m, 3H), $5.46(\mathrm{~s}, 2 \mathrm{H}), 4.05-3.98(\mathrm{~m}, 4 \mathrm{H}), 3.35-3.30(\mathrm{~m}, 4 \mathrm{H}), 2.43$ (s, 3H) ppm. ${ }^{13} \mathrm{C}$ NMR (101 MHz, $\left.\mathrm{CDCl}_{3}\right) \delta 162.4(\mathrm{C}), 161.3(\mathrm{C}), 159.5(\mathrm{C}), 150.8(\mathrm{C}), 140.9$ (C), $131.6(\mathrm{C}), 129.6(2 \times C H), 129.3(2 \times C H), 128.6(2 \times C H), 120.4(\mathrm{CH}), 117.7(\mathrm{C}), 116.4(\mathrm{C})$, $116.3(2 \times C H), 84.0(\mathrm{C}), 82.5(\mathrm{C}), 49.2(2 \times C H), 47.7(2 \times C H), 21.5(\mathrm{CH})$ ppm. HRMS: calcd for $\mathrm{C}_{24} \mathrm{H}_{23} \mathrm{~N}_{6}[\mathrm{M}+\mathrm{H}]^{+} 395.1979$, found 395.1978.

\subsection{Palladium Catalyzed Cyanation: General Procedure for Synthesis of Compounds 11-13}

A mixture of halo derivative (50 mg; 1 eq.), $\mathrm{Pd}_{2}(\mathrm{dba})_{3}\left(0.05\right.$ eq.), $\mathrm{dppf}\left(0.1\right.$ eq.), $\mathrm{Zn}(\mathrm{CN})_{2}$ ( 0.6 eq.), $\mathrm{Zn}(0.2$ eq. $)$ in eucalyptol $(2 \mathrm{~mL})$ was stirred at $140-170{ }^{\circ} \mathrm{C}$ for $26-44 \mathrm{~h}$. The reaction was followed by TLC. After completion, the reaction was cooled to room temperature, and the mixture was concentrated under vacuum. The solid obtained was purified by flash chromatography using a mixture of AcOEt/petroleum ether.

Thieno[3,2-d]pyrimidine-4-carbonitrile (11). White solid $(26 \mathrm{mg}, 56 \%){ }^{1} \mathrm{H} \mathrm{NMR}(400 \mathrm{MHz}$, $\left.\mathrm{CDCl}_{3}\right) \delta 7.69(\mathrm{~d}, J=5.5 \mathrm{~Hz}, 1 \mathrm{H}), 8.23(\mathrm{~d}, J=5.5 \mathrm{~Hz}, 1 \mathrm{H}), 9.34(\mathrm{~s}, 1 \mathrm{H})$ ppm. 13C NMR $(101$ $\mathrm{MHz}, \mathrm{CDCl} 3) \delta 114.3(\mathrm{C}), 125.0(\mathrm{CH}), 133.5(\mathrm{C}), 135.6(\mathrm{C}), 138.8(\mathrm{CH}), 154.6(\mathrm{CH}), 162.5(\mathrm{C})$ ppm. [CAS: 1057249-33-8].

Thieno[3,2-b]pyridine-7-carbonitrile (12). White solid (29 mg, 61\%) ${ }^{1} \mathrm{H} \mathrm{NMR}(400 \mathrm{MHz}$, $\left.\mathrm{CDCl}_{3}\right) \delta 8.84(\mathrm{~d}, J=4.7 \mathrm{~Hz}, 1 \mathrm{H}), 7.93(\mathrm{~d}, J=5.5 \mathrm{~Hz}, 1 \mathrm{H}), 7.67(\mathrm{~d}, J=5.5 \mathrm{~Hz}, 1 \mathrm{H}), 7.53(\mathrm{~d}$, $J=4.7 \mathrm{~Hz}, 1 \mathrm{H}) \mathrm{ppm} .{ }^{13} \mathrm{C}$ NMR $\left(101 \mathrm{MHz}, \mathrm{CDCl}_{3}\right) \delta 157.2(\mathrm{C}), 147.3(\mathrm{CH}), 133.9(\mathrm{C}), 132.5$ (CH), $125.7(\mathrm{CH}), 121.0(\mathrm{CH}), 115.2(\mathrm{C}), 114.8$ (C) ppm. [CAS: 1239505-20-4].

6-phenylthieno[2,3-b]pyrazine-7-carbonitrile (13). Brown solid (29 mg, 72\%) ${ }^{1} \mathrm{H} \mathrm{NMR}$ $\left(400 \mathrm{MHz}, \mathrm{CDCl}_{3}\right) \delta 8.79(\mathrm{~d}, J=2.4 \mathrm{~Hz}, 1 \mathrm{H}), 8.60(\mathrm{~d}, J=2.4 \mathrm{~Hz}, 1 \mathrm{H}), 8.01-7.96(\mathrm{~m}, 2 \mathrm{H})$, 7.62-7.57 (m, 3H) ppm. ${ }^{13} \mathrm{C}$ NMR (101 MHz, $\left.\mathrm{CDCl}_{3}\right) \delta 159.0(\mathrm{C}), 153.5(\mathrm{C}), 149.4(\mathrm{C}), 143.4$ $(\mathrm{CH}), 142.0(\mathrm{CH}), 131.8(\mathrm{CH}), 130.8(\mathrm{C}), 129.7(2 \times \mathrm{CH}), 128.4(2 \times \mathrm{CH}), 113.5(\mathrm{C}), 101.8(\mathrm{C})$ ppm. [CAS: 1369884-57-0].

\subsection{Hiyama Coupling: General Procedure for Synthesis of Compounds 14-21}

A mixture of halo derivative (50 mg; 1 eq.), $\mathrm{Pd}\left(\mathrm{CH}_{3} \mathrm{CN}\right)_{2} \mathrm{Cl}_{2}$ (0.05 eq.), $\mathrm{PPh}_{3}(0.15$ eq.), $\mathrm{Cs}_{2} \mathrm{CO}_{3}$ (2 eq.) in eucalyptol $(2 \mathrm{~mL})$ was stirred at $100{ }^{\circ} \mathrm{C}$ for $30-48 \mathrm{~h}$. The reaction was followed by TLC. After completion, the reaction was cooled to room temperature, and the mixture was concentrated under vacuum. The solid obtained was purified by flash chromatography using a mixture of AcOEt/petroleum ether.

7-(phenylethynyl)thieno[3,2-b]pyridine (14). Yellow solid (55 mg, 80\%), m.p. 139-141 ${ }^{\circ} \mathrm{C}$. ${ }^{1} \mathrm{H}$ NMR $\left(400 \mathrm{MHz}, \mathrm{CDCl}_{3}\right) \delta 8.69(\mathrm{~d}, J=4.8 \mathrm{~Hz}, 1 \mathrm{H}), 7.79(\mathrm{~d}, J=5.5 \mathrm{~Hz}, 1 \mathrm{H}), 7.64(\mathrm{dd}$, $J=6.3,2.7 \mathrm{~Hz}, 2 \mathrm{H}), 7.59(\mathrm{~d}, J=5.5 \mathrm{~Hz}, 1 \mathrm{H}), 7.44-7.39(\mathrm{~m}, 3 \mathrm{H}), 7.36(\mathrm{~d}, J=4.8 \mathrm{~Hz}, 1 \mathrm{H}) \mathrm{ppm}$. ${ }^{13} \mathrm{C}$ NMR (101 MHz, $\left.\mathrm{CDCl}_{3}\right) \delta 156.1(\mathrm{C}), 147.3(\mathrm{CH}), 134.9(\mathrm{C}), 132.0(2 \times \mathrm{CH}), 130.9(\mathrm{CH})$, $129.5(\mathrm{CH}), 128.6(2 \times \mathrm{CH}), 126.1(\mathrm{C}), 125.6(\mathrm{CH}), 121.9(\mathrm{C}), 120.3(\mathrm{CH}), 97.9(\mathrm{C}), 84.8(\mathrm{C}) \mathrm{ppm}$. HRMS: calcd for $\mathrm{C}_{15} \mathrm{H}_{10} \mathrm{NS}[\mathrm{M}+\mathrm{H}]^{+} 236.0528$, found 236.0528 .

7-allylthieno[3,2-b]pyridine (15). Yellow solid (27 mg, 53\%), m.p. $136-138{ }^{\circ} \mathrm{C} .{ }^{1} \mathrm{H} \mathrm{NMR}$ $\left(400 \mathrm{MHz}, \mathrm{CDCl}_{3}\right) \delta 8.63(\mathrm{~d}, J=4.9 \mathrm{~Hz}, 1 \mathrm{H}), 7.73(\mathrm{~d}, J=5.6 \mathrm{~Hz}, 1 \mathrm{H}), 7.58(\mathrm{~d}, J=5.6 \mathrm{~Hz}, 1 \mathrm{H})$, $7.20(\mathrm{~d}, J=4.9 \mathrm{~Hz}, 1 \mathrm{H}), 6.77-6.59(\mathrm{~m}, 2 \mathrm{H}), 2.05-2.03(\mathrm{~m}, 3 \mathrm{H}) \mathrm{ppm} .{ }^{13} \mathrm{C}$ NMR $(101 \mathrm{MHz}$, $\left.\mathrm{CDCl}_{3}\right) \delta 157.0(\mathrm{C}), 147.6(\mathrm{CH}), 140.1(\mathrm{C}), 133.5(\mathrm{CH}), 130.4(\mathrm{C}), 129.8(\mathrm{CH}), 127.7(\mathrm{CH}), 125.6$ $(\mathrm{CH}), 115.3(\mathrm{CH}), 19.0(\mathrm{CH})$ ppm. HRMS: calcd for $\mathrm{C}_{10} \mathrm{H}_{10} \mathrm{NS}[\mathrm{M}+\mathrm{H}]^{+} 176.0528$, found 176.0530 .

7-((4-(trifluoromethyl)phenyl)ethynyl)thieno[3,2-b]pyridine (16). Yellow solid (45 mg, 50\%), m.p. $161-163{ }^{\circ} \mathrm{C} .{ }^{1} \mathrm{H}$ NMR $(400 \mathrm{MHz}, \mathrm{CDCl} 3) \delta 8.71(\mathrm{~d}, J=4.8 \mathrm{~Hz}, 1 \mathrm{H}), 7.81(\mathrm{~d}, J=5.5$ $\mathrm{Hz}, 1 \mathrm{H}), 7.74(\mathrm{~d}, J=8.1 \mathrm{~Hz}, 2 \mathrm{H}), 7.67(\mathrm{~d}, J=8.2 \mathrm{~Hz}, 2 \mathrm{H}), 7.61(\mathrm{~d}, J=5.6 \mathrm{~Hz}, 1 \mathrm{H}), 7.38(\mathrm{~d}$, $J=4.8 \mathrm{~Hz}, 1 \mathrm{H})$ ppm.

${ }^{13} \mathrm{C} \mathrm{NMR}\left(101 \mathrm{MHz}, \mathrm{CDCl}_{3}\right) \delta 156.2(\mathrm{C}), 147.3(\mathrm{CH}), 134.9(\mathrm{C}), 132.3(3 \times \mathrm{CH}), 131.3$ (C), $131.0(\mathrm{CH}), 125.7(2 \times C H), 125.5(\mathrm{CH}), 125.3(\mathrm{C}), 122.4(\mathrm{C}), 120.4(\mathrm{C}), 95.9(\mathrm{C}), 86.8(\mathrm{C})$ ppm. ${ }^{19} \mathrm{~F} \mathrm{NMR}\left(376 \mathrm{MHz}, \mathrm{CDCl}_{3}\right) \delta-62.95$ ppm. HRMS: calcd for $\mathrm{C}_{16} \mathrm{H}_{9} \mathrm{~F}_{3} \mathrm{NS}[\mathrm{M}+\mathrm{H}]^{+}$ 304.0402, found 304.0403.

7-(furan-3-ylethynyl)thieno[3,2-b]pyridine (17). White solid (46 mg, 69\%), m.p. 103-105 ${ }^{\circ} \mathrm{C}$. ${ }^{1} \mathrm{H} \mathrm{NMR}\left(400 \mathrm{MHz} \mathrm{CDCl}_{3}\right) \delta 8.67(\mathrm{~d}, J=4.8 \mathrm{~Hz}, 1 \mathrm{H}), 7.83-7.76(\mathrm{~m}, 2 \mathrm{H}), 7.59(\mathrm{~d}, J=5.5 \mathrm{~Hz}$, 
$1 \mathrm{H}), 7.46(\mathrm{t}, J=1.7 \mathrm{~Hz}, 1 \mathrm{H}), 7.32(\mathrm{~d}, J=4.8 \mathrm{~Hz}, 1 \mathrm{H}), 6.61(\mathrm{~d}, J=1.7 \mathrm{~Hz}, 1 \mathrm{H}) \mathrm{ppm} .{ }^{13} \mathrm{C}$ NMR $\left(101 \mathrm{MHz}, \mathrm{CDCl}_{3}\right) \delta 156.0(\mathrm{C}), 147.2(\mathrm{CH}), 146.8(\mathrm{CH}), 143.3(\mathrm{CH}), 134.6(\mathrm{C}), 130.9(\mathrm{CH})$, 126.1 (C), $125.5(\mathrm{CH}), 120.2(\mathrm{CH}), 112.5(\mathrm{CH}), 106.7(\mathrm{C}), 89.3(\mathrm{C}), 86.7(\mathrm{C})$ ppm. HRMS: calcd for $\mathrm{C}_{13} \mathrm{H}_{8} \mathrm{NOS}[\mathrm{M}+\mathrm{H}]^{+}$226.0321, found 226.0322 .

7-((4-methoxyphenyl)ethynyl)thieno[3,2-b]pyridine (18). White solid (63 mg, 81\%), m.p. $142-144{ }^{\circ} \mathrm{C} .{ }^{1} \mathrm{H}$ NMR $\left(400 \mathrm{MHz}, \mathrm{CDCl}_{3}\right) \delta 8.66(\mathrm{~d}, J=4.8 \mathrm{~Hz}, 1 \mathrm{H}), 7.76(\mathrm{~d}, J=5.5 \mathrm{~Hz}, 1 \mathrm{H})$, $7.60-7.53(\mathrm{~m}, 3 \mathrm{H}), 7.31(\mathrm{~d}, J=4.8 \mathrm{~Hz}, 1 \mathrm{H}), 6.91(\mathrm{~d}, J=8.8 \mathrm{~Hz}, 2 \mathrm{H}), 3.83(\mathrm{~s}, 3 \mathrm{H}) \mathrm{ppm} .{ }^{13} \mathrm{C}$ NMR (101 MHz, CDCl $) \delta 160.6(\mathrm{C}), 156.0(\mathrm{C}), 147.2(\mathrm{CH}), 134.8(\mathrm{C}), 133.7(2 \times \mathrm{CH}), 130.7$ $(\mathrm{CH}), 126.5(\mathrm{C}), 125.6(\mathrm{CH}), 120.0(\mathrm{CH}), 114.2(2 \times \mathrm{CH}), 113.9(\mathrm{C}), 98.3(\mathrm{C}), 83.9(\mathrm{C}), 55.4(\mathrm{CH})$ ppm. HRMS: calcd for $\mathrm{C}_{16} \mathrm{H}_{12} \mathrm{NOS}[\mathrm{M}+\mathrm{H}]^{+} 266.0634$, found 266.0638 .

7-(thiophen-3-ylethynyl)thieno[3,2-b]pyridine (19). White solid (36 mg, 51\%), m.p. 99-101 ${ }^{\circ} \mathrm{C}$. ${ }^{1} \mathrm{H}$ NMR $\left(400 \mathrm{MHz}, \mathrm{CDCl}_{3}\right) \delta 8.67(\mathrm{~d}, J=4.8 \mathrm{~Hz}, 1 \mathrm{H}), 7.77(\mathrm{~d}, J=5.5 \mathrm{~Hz}, 1 \mathrm{H}), 7.68(\mathrm{dd}, J=2.9$, $1.1 \mathrm{~Hz}, 1 \mathrm{H}), 7.58(\mathrm{~d}, J=5.5 \mathrm{~Hz}, 1 \mathrm{H}), 7.37-7.31(\mathrm{~m}, 2 \mathrm{H}), 7.28(\mathrm{dd}, J=5.0,1.0 \mathrm{~Hz}, 1 \mathrm{H}) \mathrm{ppm} .{ }^{13} \mathrm{C}$ NMR (101 MHz, CDCl 3 ) $\delta 156.1(\mathrm{C}), 147.2(\mathrm{CH}), 134.8(\mathrm{C}), 130.8(\mathrm{CH}), 130.6(\mathrm{CH}), 129.9(\mathrm{CH})$, $126.1(\mathrm{C}), 125.9(\mathrm{CH}), 125.6(\mathrm{CH}), 121.0(\mathrm{C}), 120.2(\mathrm{CH}), 93.0(\mathrm{C}), 84.5(\mathrm{C})$ ppm. HRMS: calcd for $\mathrm{C}_{13} \mathrm{H}_{8} \mathrm{NS}_{2}[\mathrm{M}+\mathrm{H}]^{+}$242.0093, found 242.0094 .

4-(phenylethynyl)furo[3,2-c]pyridine (20). White solid (21 mg, 30\%) ${ }^{1} \mathrm{H}$ NMR $(400 \mathrm{MHz}$, $\left.\mathrm{CDCl}_{3}\right) \delta 8.51(\mathrm{~d}, J=5.7 \mathrm{~Hz}, 1 \mathrm{H}), 7.70(\mathrm{~d}, J=1.9 \mathrm{~Hz}, 1 \mathrm{H}), 7.66(\mathrm{dd}, J=6.2,2.7 \mathrm{~Hz}, 2 \mathrm{H})$, 7.43-7.38 (m, 4H), $7.03(\mathrm{~s}, 1 \mathrm{H}) \mathrm{ppm} .{ }^{13} \mathrm{C} \mathrm{NMR}\left(101 \mathrm{MHz}, \mathrm{CDCl}_{3}\right) \delta 159.2(\mathrm{C}), 146.0(\mathrm{CH})$, $144.9(\mathrm{CH}), 137.5(\mathrm{C}), 132.1(2 \times \mathrm{CH}), 129.2(\mathrm{CH}), 128.5(2 \times \mathrm{CH}), 127.0(\mathrm{C}), 122.2(\mathrm{C}), 106.9$ $(\mathrm{CH}), 105.5(\mathrm{CH}), 92.7(\mathrm{C}), 86.5(\mathrm{C}) \mathrm{ppm}$. [CAS: 2098141-91-2].

4-((4-methoxyphenyl)ethynyl)furo[3,2-c]pyridine (21). Yellow solid (39 mg, 48\%) ${ }^{1} \mathrm{H}$ NMR $(400 \mathrm{MHz}, \mathrm{CDCl} 3) \delta 8.49(\mathrm{~d}, J=5.7 \mathrm{~Hz}, 1 \mathrm{H}), 7.69(\mathrm{~d}, J=2.2 \mathrm{~Hz}, 1 \mathrm{H}), 7.61-7.57(\mathrm{~m}, 2 \mathrm{H})$, $7.40(\mathrm{~d}, J=6.6 \mathrm{~Hz}, 1 \mathrm{H}), 7.01(\mathrm{dd}, J=2.2,0.9 \mathrm{~Hz}, 1 \mathrm{H}), 6.93-6.89(\mathrm{~m}, 2 \mathrm{H}), 3.84(\mathrm{~s}, 3 \mathrm{H}) \mathrm{ppm}$. ${ }^{13} \mathrm{C}$ NMR $\left(101 \mathrm{MHz}, \mathrm{CDCl}_{3}\right) \delta 160.4(\mathrm{C}), 159.2(\mathrm{C}), 145.8(\mathrm{CH}), 144.8(\mathrm{CH}), 137.9(\mathrm{C}), 133.7$ $(2 \times C H), 126.7(\mathrm{C}), 114.2(\mathrm{C}), 114.1(2 \times C H), 106.6(\mathrm{CH}), 105.6(\mathrm{CH}), 93.1(\mathrm{C}), 85.5(\mathrm{C}), 55.3$ (CH) $\mathrm{ppm}$.

\section{Conclusions}

Simple conditions to generate several $\mathrm{O}, \mathrm{S}, \mathrm{N}$-Heterocycles by Hiyama coupling, cyanation and multicomponent reactions, which may show interesting biological properties, have been presented in this paper. Methods of preparation were optimized using eucalyptol as a biobased solvent.

Author Contributions: Conceptualization and methodology, J.F.C. and S.B.-R.; investigation and data curation, J.F.C.; Performance of the experiments, J.F.C.; Analysis of some products, V.F.; writingoriginal draft preparation, J.F.C.; writing - review and editing, S.B.-R.; supervision, project administration, and funding acquisition, S.B.-R. All authors have read and agreed to the published version of the manuscript.

Funding: This research received no external funding.

Data Availability Statement: The data presented in this study are available on request from the corresponding author.

Conflicts of Interest: The authors declare no conflict of interest.

\section{References}

1. Henderson, R.K.; Jiménez-González, C.; Constable, D.J.C.; Alston, S.R.; Inglis, G.G.A.; Fisher, G.; Sherwood, J.; Binks, S.P.; Curzons, A.D. Expanding GSK's solvent selection guide-Embedding sustainability into solvent selection starting at medicinal chemistry. Green Chem. 2011, 13, 854-862. [CrossRef]

2. Clarke, C.J.; Tu, W.-C.; Levers, O.; Bröhl, A.; Hallett, J.P. Green and Sustainable Solvents in Chemical Processes. Chem. Rev. 2018, 118, 747-800. [CrossRef]

3. Capello, C.; Fischer, U.; Hungerbühler, K. What is a green solvent? A comprehensive framework for the environmental assessment of solvents. Green Chem. 2007, 9, 927-934. [CrossRef] 
4. Alfonsi, K.; Colberg, J.; Dunn, P.J.; Fevig, T.; Jennings, S.; Johnson, T.A.; Kleine, H.P.; Knight, C.; Nagy, M.A.; Perry, D.A.; et al. Green chemistry tools to influence a medicinal chemistry and research chemistry based organization. Green Chem. 2008, 10, 31-36. [CrossRef]

5. Prat, D.; Pardigon, O.; Flemming, H.W.; Letestu, S.; Ducandas, V.; Isnard, P.; Guntrum, E.; Senac, T.; Ruisseau, S.; Cruciani, P.; et al. Sanofi's Solvent Selection Guide: A Step Toward More Sustainable Processes. Org. Process Res. Dev. 2013, 17, $1517-1525$. [CrossRef]

6. Diorazio, L.J.; Hose, D.R.J.; Adlington, N.K. Toward a More Holistic Framework for Solvent Selection. Org. Process Res. Dev. 2016, 20, 760-773. [CrossRef]

7. Campos, J.F.; Berteina-Raboin, S. Greener Synthesis of Nitrogen-Containing Heterocycles in Water, PEG and Bio-Based Solvents. Catalysts 2020, 10, 429. [CrossRef]

8. Campos, J.F.; Pacheco-Benichou, A.; Fruit, C.; Besson, T.; Berteina-Raboin, S. Synthesis of Benzo-Fused 11H-Pyrido[2,1-b]quinazolin-11ones by a Buchwald-Hartwig Coupling/Pyridine Dearomatization Sequence in Eucalyptol. Synthesis 2020, 52, 3071-3076. [CrossRef]

9. Fresneau, N.; Hiebel, M.-A.; Agrofoglio, L.A.; Berteina-Raboin, S. Efficient Synthesis of Unprotected C-5-Aryl/Heteroaryl-2' deoxyuridine via a Suzuki-Miyaura Reaction in Aqueous Media. Molecules 2012, 17, 14409. [CrossRef]

10. Fresneau, N.; Hiebel, M.-A.; Agrofoglio, L.A.; Berteina-Raboin, S. One-pot Sonogashira-cyclization protocol to obtain substituted furopyrimidine nucleosides in aqueous conditions. Tetrahedron Lett. 2012, 53, 1760-1763. [CrossRef]

11. Hiebel, M.-A.; Fall, Y.; Scherrmann, M.-C.; Berteina-Raboin, S. Straightforward Synthesis of Various 2,3-Diarylimidazo[1,2a]pyridines in PEG400 Medium through One-Pot Condensation and C-H Arylation. Eur. J. Org. Chem. 2014, 21, 4643-4650. [CrossRef]

12. Hiebel, M.-A.; Berteina-Raboin, S. Iodine-catalyzed regioselective sulfenylation of imidazoheterocycles in PEG400. Green Chem. 2015, 17, 937-944. [CrossRef]

13. Dumonteil, G.; Hiebel, M.-A.; Scherrmann, M.-C.; Berteina-Raboin, S. Iodine-catalyzed formation of substituted 2aminobenzothiazole derivatives in PEG400. RSC Adv. 2016, 6, 73517-73521. [CrossRef]

14. Campos, J.F.; Loubidi, M.; Scherrmann, M.-C.; Berteina-Raboin, S. A Greener and Efficient Method for Nucleophilic Aromatic Substitution of Nitrogen-Containing Fused Heterocycles. Molecules 2018, 23, 684. [CrossRef]

15. Campos, J.F.; Scherrmann, M.-C.; Berteina-Raboin, S. Eucalyptol: A new solvent for the synthesis of heterocycles containing oxygen, sulfur and nitrogen. Green Chem. 2019, 21, 1531-1539. [CrossRef]

16. Campos, J.F.; Berteina-Raboin, S. Eucalyptol as bio-based solvent for Migita-Kosugi-Stille coupling reaction on O,S,N-Heterocycles. Catal. Today 2020, 358, 138-142. [CrossRef]

17. Campos, J.F.; Berteina-Raboin, S. Eucalyptol as a Bio-Based Solvent for Buchwald-Hartwig Reaction on O,S,N-Heterocycles. Catalysts 2019, 9, 840. [CrossRef]

18. Lombardino, J.G.; Lowe, J.A., III. A Guide to drug discovery: The Role of Medicinal Chemist in Drug Discovery-Then and Now. Nat. Rev. Drug Discov. 2004, 3, 853-862. [CrossRef]

19. Hayashi, Y. Pot economy and one-pot synthesis. Chem. Sci. 2016, 7, 866-880. [CrossRef] [PubMed]

20. Jiang, B.; Rajale, T.; Wever, W.; Tu, S.-J.; Li, G. Multicomponent Reactions for the Synthesis of Heterocycles. Chem. Asian J. 2010, 5, 2318-2335. [CrossRef]

21. Malinakova, H.C. Recent advances in the discovery and design of multicomponent reactions for the generation of small-molecule libraries. Rep. Org. Chem. 2015, 5, 75-90. [CrossRef]

22. Altaf, A.A.; Shahzad, A.; Gul, Z.; Rasool, N.; Badshah, A.; Lal, B.; Khan, E. A Review on the Medicinal Importance of Pyridine Derivatives. JDDMC 2015, 1, 1-11. [CrossRef]

23. Fuentes, L.; Vaquero, J.J.; Soto, J.L. Heterocycle synthesis. XVI. Reaction of malononitrile with benzylidenemalononitriles in presence of amines. AN QUIM C-ORG BIOQ 1980, 76, 68-69.

24. Raghukumar, V.; Thirumalai, D.; Ramakrishnan, V.T.; Karunakara, V.; Ramamurthy, P. Synthesis of nicotinonitrile derivatives as a new class of Non-linear optical materials. Tetrahedron 2003, 59, 3761-3768. [CrossRef]

25. Sarkar, S.; Das, D.K.; Khan, A.T. Synthesis of fully-substituted pyridines and dihydropyridines in a highly chemoselective manner utilizing a multicomponent reaction (MCR) strategy. RSC Adv. 2014, 4, 53752-53760. [CrossRef]

26. Rosenmund, K.W.; Struck, E. Das am Ringkohlenstoff gebundene Halogen und sein Ersatz durch andere Substituenten. I. Mitteilung: Ersatz des Halogens durch die Carboxylgruppe. Chem. Ber. 1919, 52, 1749-1756. [CrossRef]

27. Pongratz, A. Untersuchungen über Perylen und seine Derivate. Monatsh. Chem. 1927, 48, 585-591. [CrossRef]

28. von Braun, J.; Manz, G. Fluoranthen und seine Derivate. III. Mitteilung. Liebigs Ann. Chem. 1931, 488, 111-126. [CrossRef]

29. Connor, J.A.; Leeming, S.W.; Price, R. Influence of substrate structure on copper(I)-assisted cyanide substitution in aryl halides. J. Chem. Soc. Perkin Trans. 1990, 1, 1127-1132. [CrossRef]

30. Ellis, G.P.; Romney-Alexander, T.M. Cyanation of aromatic halides. Chem. Rev. 1987, 87, 779-794. [CrossRef]

31. Campos, J.F.; Queiroz, M.-J.R.P.; Berteina-Raboin, S. The first catalytic direct C-H arylation on C2 and C3 of thiophene ring applied to thieno-pyridines, -pyrimidines and -pyrazines. Catalysts 2018, 8, 137. [CrossRef]

32. Campos, J.F.; Queiroz, M.-J.R.P.; Berteina-Raboin, S. Synthesis of new thieno[3,2- $b]$ pyridines and thieno[3,2- $b]$ pyrazines by palladium cross-coupling. ChemistrySelect 2017, 24, 6945-6948. [CrossRef]

33. Schareina, T.; Zapf, A.; Beller, M. An environmentally benign procedure for the Cu-catalyzed cyanation of aryl bromides. Tetrahedron Lett. 2005, 15, 2585-2588. [CrossRef] 
34. Schareina, T.; Zapf, A.; Mägerlein, W.; Müller, N.; Beller, M. A State-of-the-Art Cyanation of Aryl Bromides: A Novel and Versatile Copper Catalyst System Inspired by Nature. Chem. Eur. J. 2007, 21, 6249-6254. [CrossRef]

35. Schareina, T.; Zapf, A.; Cotté, A.; Müller, N.; Beller, M. A Bio-inspired Copper Catalyst System for Practical Catalytic Cyanation of Aryl Bromides. Synthesis 2008, 20, 3351-3355. [CrossRef]

36. Schareina, T.; Zapf, A.; Mägerlein, W.; Müller, N.; Beller, M. Copper-Catalyzed Cyanation of Heteroaryl Bromides: A Novel and Versatile Catalyst System Inspired by Nature. Synlett 2007, 4, 555-558. [CrossRef]

37. Anbarasan, P.; Schareina, T.; Beller, M. Recent developments and perspectives in palladium-catalyzed cyanation of aryl halides: Synthesis of benzonitriles. Chem. Soc. Rev. 2011, 40, 5049-5067. [CrossRef] [PubMed]

38. Montel, F.; Lamberth, C.; Jung, P.M.J. First synthesis of 7-amido-[1,2,4]triazolo[1,5-a]pyrimidines using halogen-metal exchange. Tetrahedron 2008, 27, 6372-6376. [CrossRef]

39. Miyashita, A.; Suzuki, Y.; Ohta, K.; Higashino, T. Preparation of Heteroarenecarbonitriles by Reaction of Haloheteroarenes with Potassium Cyanide Catalyzed by Sodium p-Toluenesulfinate. Heterocycles 1994, 39, 345-356. [CrossRef]

40. Ballini, R.; Belderrain, T.R.; Bruneau, C.; Cokoja, M.; Dong, D. Science of Synthesis: C-1 Building Blocks in Organic Synthesis Vol. 2: Alkenations, Cross Couplings, Insertions, Substitutions, and Halomethylations (English Edition); Thieme Publishers: New York, NY, USA; Georg Thieme Verlag KG: Stuttgart, Germany, 2014; ISBN 9783131751317.

41. Schareina, T.; Zapf, A.; Beller, M. Potassium hexacyanoferrate(ii)—A new cyanating agent for the palladium-catalyzed cyanation of aryl halides. Chem. Commun. 2004, 1388-1389. [CrossRef] [PubMed]

42. Li, J.J. Hiyama Cross-Coupling Reaction in: Name Reactions; Springer: Berlin/Heidelberg, Germany, 2003; pp. 187-188. [CrossRef]

43. Li, J.-H.; Deng, C.-L.; Liu, W.-J.; Xie, Y.-X. Pd(OAc) $)_{2} / \mathrm{DABCO}$ as an Inexpensive and Efficient Catalytic System for HiyamaCross-Coupling Reactions of Aryl Halides with Aryltrimethoxysilanes. Synthesis 2005, 18, 3039-3044. [CrossRef]

44. Molander, G.A.; Iannazzo, L. Palladium-Catalyzed Hiyama Cross-Coupling of Aryltrifluorosilanes with Aryl and Heteroaryl Chlorides. J. Org. Chem. 2011, 76, 9102-9108. [CrossRef] [PubMed]

45. Monguchi, Y.; Yanase, T.; Mori, S.; Sajiki, H. A Practical Protocol for the Hiyama Cross-Coupling Reaction Catalyzed by Palladium on Carbon. Synthesis 2013, 45, 40-44. [CrossRef]

46. Raders, S.M.; Kingston, J.V.; Verkade, J.G. Advantageous Use of ${ }^{t} \mathrm{Bu}_{2} \mathrm{P}-\mathrm{N}=\mathrm{P}\left(\mathrm{iBuNCH}_{2} \mathrm{CH}_{2}\right)_{3} \mathrm{~N}$ in the Hiyama Coupling of Aryl Bromides and Chlorides. J. Org. Chem. 2010, 75, 1744-1747. [CrossRef] [PubMed]

47. Srimani, D.; Bej, A.; Sarkar, A. Palladium Nanoparticle Catalyzed Hiyama Coupling Reaction of Benzyl Halides. J. Org. Chem. 2010, 75, 4296-4299. [CrossRef] 